\title{
Proteger y defender la Manga del Mar Menor: estudio histórico- arqueológico de la Torre de San Miguel del Estacio y la Torre de la Encañizada
}

\section{Benjamín Cutillas Victoria}

Universidad de Murcia, Murcia, España, benjamincutillas@yahoo.es

\begin{abstract}
During the Sixteenth century, the North African pirates' attacks hit peninsular coasts, including the southeast peninsular. In this situation, La Manga del Mar Menor was presented like a paramount position which ought to be defended, with the aim of avoid the pirates' entrance in the Mar Menor, from where they could attack the productive fields of Cartagena and Murcia. Moreover, the pirates have conquered Isla Grosa, and it has become a point of watery from where they can operate.

Therefore, two fortified towers were built in order to protect these coasts: the tower of San Miguel del Estacio, from where they can criticize harshly the ships which could approach to Isla Grosa; and the tower of La Encañizada, erected to defend the fertile weir's fishing area and to protect the only channel which communicated the Mar Menor and the Mediterranean Sea.
\end{abstract}

Keywords: La Manga del Mar Menor, watchtower, fishing, piracy.

\section{Introducción}

La Manga del Mar Menor fue utilizada a modo de fortificación adelantada, pues, el hecho de su defensa no sólo pretendía ocuparse de este cordón litoral de 22 kilómetros de longitud, sino que buscaba evitar el ataque directo al centro de lo que hoy es la Región de Murcia: la invasión de la costa oeste del Mar Menor con la que poder acceder a los caminos de Cartagena, Murcia e, incluso, al de Andalucía.

Además, hubo un factor que determinó la urgencia del proceso de fortificación: el establecimiento en la Isla Grosa de una escala pirata gracias a la existencia de un punto de aguada y al abrigo natural que proporcionaba como fondeadero, utilizado al menos desde época fenicia, como atestigua el pecio fenicio del Bajo de la Campana datado en el siglo VII a.C. (Berrocal, Pérez, 2010).
Con ese fin, dos torres se edificaron en la parte norte del cordón litoral, respondiendo tanto a necesidades geoestratégicas como económicas, ya que, como veremos, esta zona ha mantenido una ocupación humana continua con el fin principal de explotar los recursos marítimos que afloran abundantemente en esta parte norte, lo que influía en la importancia de su fortificación.

Como hitos clave de esta etapa histórica, el objetivo de este trabajo es realizar una revisión de conjunto de ambas torres, relacionando estos resultados con nuevas perspectivas de análisis, como es el caso de la geoestrategia de su ubicación, cálculos de visibilidad y el análisis arqueológico de los vestigios que aún quedan como testigos de lo que, otrora, fueron las grandes Torres del Estacio y de la Encañizada. 
2. Tiempos hostiles, tiempos de fortificación: la respuesta ante una nueva amenaza

Los ataques berberiscos a la costa murciana constituyeron un fenómeno que marcó la vida y las dinámicas tanto de las regiones litorales como de las prelitorales durante los siglos XVI y XVII. Estas incursiones enemigas que se venían produciendo desde inicios de siglo, plantaron la semilla del miedo y la inseguridad entre concejos y población sobre lo que podía llegar desde el mar, lo que desembocó en diversos planes y estrategias para la construcción de infraestructuras defensivas capaces de plantar cara al enemigo, proteger a los habitantes y defender el interior desde la costa.

Pese a que los planes de fortificación de la costa murciana más conocidos fueron los realizados a partir de 1570 por Vespasiano Gonzaga y Juan Bautista Antonelli (Cámara, 1991) para contrarrestar los ataques berberiscos que desde mediados del siglo XVI se producían con mayor frecuencia, la inseguridad existente desde inicios del mismo siglo ya había obligado a tomar medidas aisladas para la zona del Mar Menor a cargo del Concejo de Murcia.

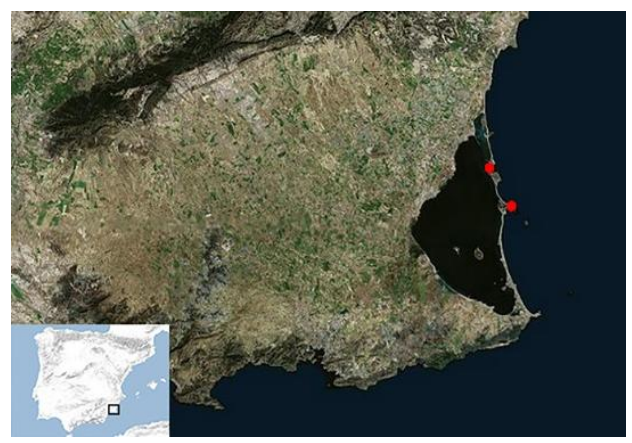

Fig. 1- Situación de la Manga del Mar Menor y los campos de Cartagena y Murcia. En rojo, las torres objeto de estudio.

Así, encontramos en 1526 la declaración del Concejo de Murcia por boca de Alonso Pacheco Arroniz explicando a los reyes que las torres eran necesarias ya que "habiéndolas estarían los pescadores seguros de los moros porque acaece muchas veces llevarse personas que estaban pescando en la Albufera" (Jiménez, 1984). Dicha ejecutoria continúa manifestando la necesidad de "rehacer en la dicha Albufera dos torres en unas peñas que están en medio de ella... y otra para la guarda del pescado que en la dicha Albufera se pesca" (Jiménez, 1984). Si bien podemos identificar la última construcción citada con la Torre de la Encañizada, parece más difícil averiguar con certeza algo sobre las otras dos torres, cuyos vestigios no han sido documentados y que, probablemente, se tratara de estructuras sitas sobre las elevaciones de Monte Blanco, El Pedruchillo o similares.

De esta forma, podemos advertir una primera fase de fortificación previa a la situación de amenaza que tuvo lugar en las últimas décadas del siglo XVI a causa de la pérdida de posesiones en el Norte de África y de la presencia cada vez más frecuente de las Armadas protestantes en las aguas del Mediterráneo (Ruiz, 1997). De esta forma, la emergente Torre de la Encañizada, levantada con el fin de proteger la próspera actividad pesquera que se desarrollaba en las Encañizadas así como a los pescadores que en ella trabajaban, se encajaba en el tejido defensivo conformado junto a otras torres que quedaban en pie en la ribera del Mar Menor de origen medieval, como podían ser las de Torre Pacheco o Los Alcázares, o el germen del actual Monasterio de San Ginés de la Jara (Jiménez, 1981).

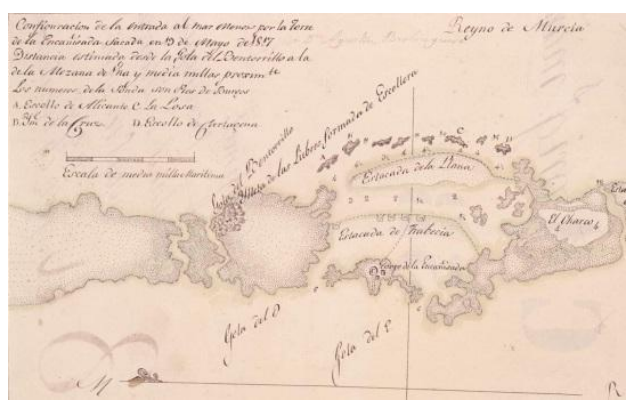

Fig. 2- Configuración de la zona de las Encañizadas en 1817 con la torre erigida cerrando la entrada al Mar Menor. AGRM.

Pese a la nueva torre construida, los ataques berberiscos se incrementaron y un nuevo factor determinante entro en escena para ambos bandos: la ocupación de Isla Grosa por los piratas y su establecimiento como un punto de aguada y óptimo fondeadero. 


\section{Reacciones centrales, medidas generales: el cordón de piedra}

Como ya hemos mencionado, la pérdida de posiciones norteafricanas como la de Túnez y la bancarrota de 1576 (Ruiz, 1997) llevaron a la monarquía hispánica a plantearse firmemente la protección de las costas peninsulares, puesto que el sistema de defensa de escalones superpuestos (Ruiz, 1995) en el que las torres litorales formaban esa tercera barrera a superar, había sufrido brechas en sus dos primeras líneas: las posiciones en la costa norteafricana y las flotas guardacostas.

Tras los informes de Gonzaga y Antonelli, se proyectaron para el litoral murciano un total de 36 torres -en otros informes se habla de 34 (Cámara, 1991)- de las que 4 se levantarían en el término de Murcia: la de San Miguel del Estacio, la del Pinatar, la del Puerto de laOlinera (¿Olvera?) y la de la "Cañizada" (Cámara, 1991). Esta última figura en esta lista por la reparación que debía hacerse en ella, como deja ver la sesión del Concejo el 25 de enero de 1578 en la que se encarga al maestro albañil Alonso Buendía que revoque toda la torre, la escalera, el pretil, asiente el enlucido y utilice agua dulce en la obra (Jiménez, 1981). No obstante, y pese al impulso inicial de los trabajos en algunas de las torres, no todas se iniciaron, quedando algunas sin edificar o retrasando su construcción hasta la última década de siglo, como la del Estacio o Portmán, o incluso hasta inicios del siglo XVII, como la Torre del Pinatar, que no fue iniciada hasta 1602. Además, más allá de los propios trabajos de erección de las estructuras defensivas, se abrían nuevos problemas como los gastos de mantenimiento de las torres o los costes del equipamiento material y humano de la guarnición, los cuales recaían sobre la población a través de un impuesto extraordinario sobre el pescado y la ganadería. En el caso de la Torre de la Encañizada también recaían sobre el arrendador de la almadraba (Ruiz, 1997).

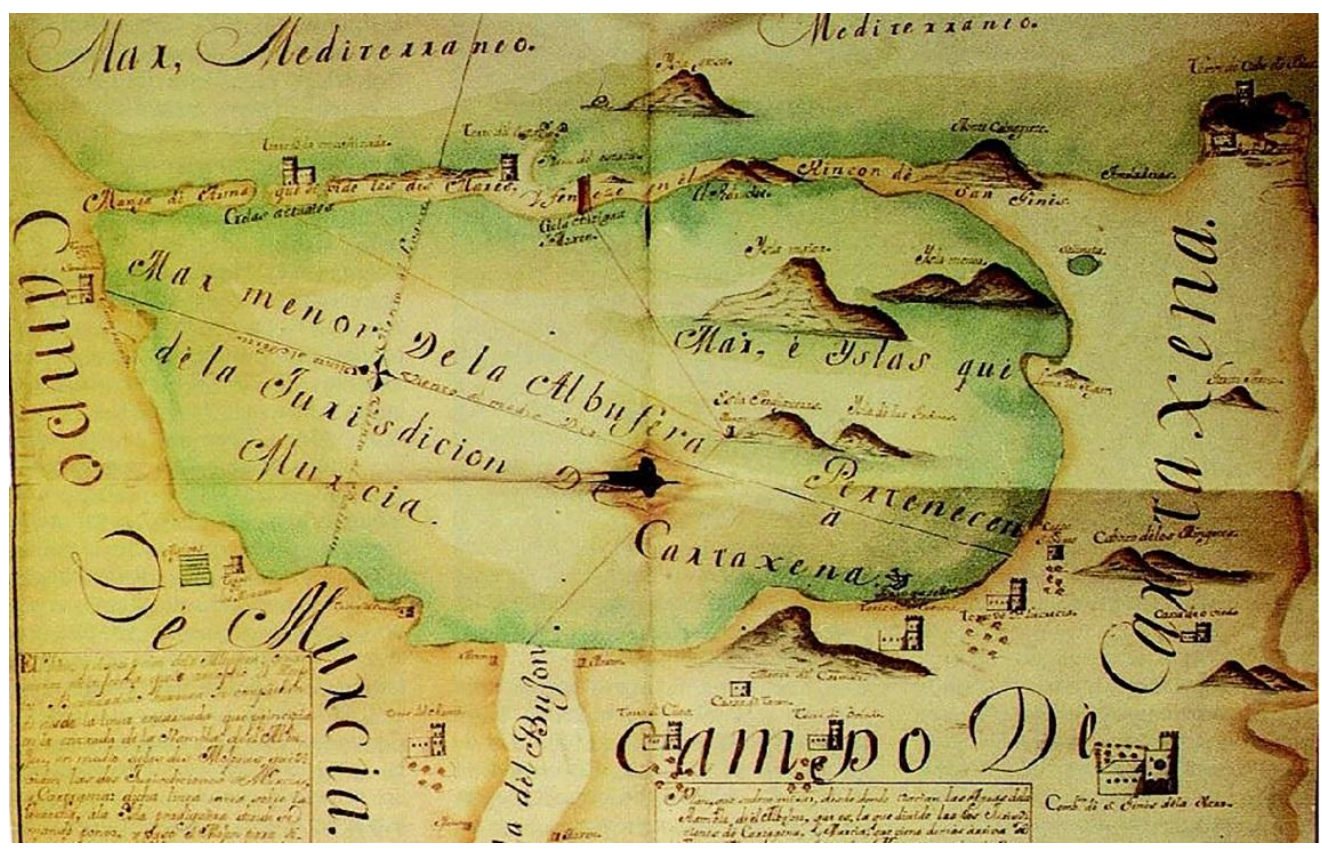

Fig. 3- Plano del Mar Menor o Albufera de Murcia en la que se pueden apreciar la torre de la Encañizada y la de San Miguel del Estacio. Anónimo. (Baragaño, Alonso, 1992).

Sin embargo, y pese a los esfuerzos invertidos, el gran problema continuó siendo la ineficacia del sistema. Torres como las de la Encañizada,
Cabo de Palos o Terreros Blancos fueron ocupadas, mientras que las de Cope y Águilas habían sido destruidas a principios del siglo 
XVII, haciendo visibles los problemas de este sistema de fortificación que defendía el sureste peninsular. Problemas que se veían agravados por la poca población, la falta de medios y un escaso interés de la Corona frente a la urgencia de acudir a otros lugares (Ruiz, 1995), encontrándose muchas de estas con períodos de abandono o de escasa guarnición.

No obstante, todas ellas fueron reparadas y continuaron funcionando hasta el siglo XVIII, aguantando las diversas embestidas enemigas $\mathrm{y}$ actuando como puntos adelantados de defensa del litoral que protegían -o más bien intentaban proteger- grandes extensiones de territorio litoral y prelitoral.

\section{Proteger la Manga del Mar Menor}

Pese a la construcción al sur de la Manga del Mar Menor de la Torre de San Antonio de Cabo de Palos en 1580, este cordón litoral se encontraba con un vacío en cuanto a protección que lo hacía un objetivo fácil, especialmente en lo referido al saqueo de la población asentada en ella y dedicada a tareas de deforestación, ganaderas, y, principalmente, a la pesca (PérezRuzafa et alii, 1987).

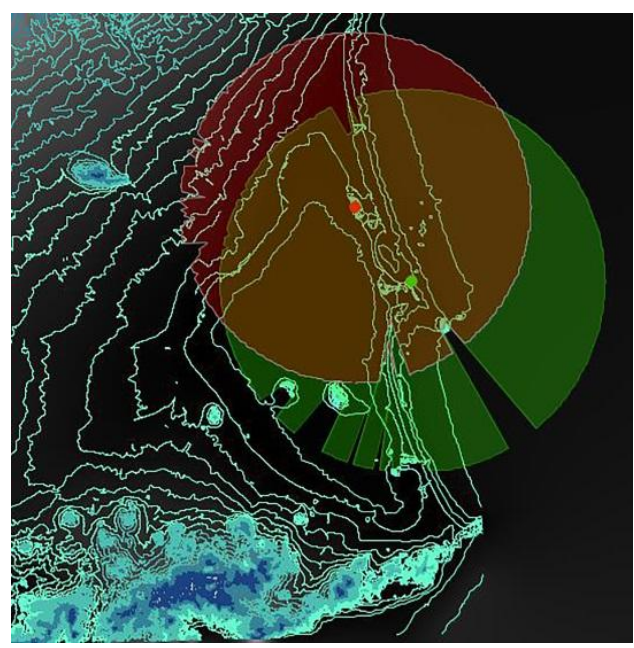

Fig.4- Cálculos de visibilidad de la Torre de San Miguel (verde) y de la Torre de la Encañizada (rojo). Radio visión utilizado: 10.000 metros.

Podemos concluir con estos datos que, a lo largo de la costa mediterránea de la Manga, se encuentran diversos fondeaderos óptimos que permitían no sólo proteger las naves ante un temporal o cargar agua dulce en Isla Grosa o en la propia barra litoral, sino la disponibilidad de hacerse con recursos alimentarios disponibles fácilmente saqueables ante una población escasa y casi huérfana de protección.

Con el fin de evitar esa útil parada para las flotas enemigas en la parte más al norte de la Manga (Fig. 4), y evitar así la instalación de una escala pirata en Isla Grosa, se proyectó la reforma y construcción de las Torres de la Encañizada y de San Miguel del Estacio, las cuales fueron edificadas a tiempo de resistir ante el enemigo pese ser atacadas e, incluso, ocupadas.

\subsection{La Torre de la Encañizada: pesca y puerta del Mar Menor}

Como ya hemos visto, la Torre de la Encañizada fue construida en torno al año 1526 en la zona de Las Encañizadas, nombre de la técnica de pesca artesanal utilizada tradicionalmente en lagunas litorales y que da nombre al lugar.

Sin duda, el valor de la zona y la riqueza en recursos pesqueros procedentes de las encañizadas así como la importante cabaña ganadera que podía pastar en los terrenos colindantes, convertía esta región del Mar Menor en un foco de producción importante. A ello debemos sumar la geoestrategia de esta zona, pues estamos ante el único paso marítimo natural al Mar Menor desde al Mar Mediterráneo (Fig.2).

Si bien es cierto que el calado de estas golas naturales no era muy profundo, podía permitir el paso de pequeños barcos y barcazas procedentes de flotas mayores que podían fondear tranquilamente en lugares cercanos, como al amparo de la Punta del Pudrimel, un poco más al sur de Las Encañizadas y cuyo suelo de arena facilitaba la función de fondeo.

Además de motivos económicos y estratégicos, la vigilancia del entorno desde un punto elevado era muy buena, pues al no existir montículos ni elevaciones cercanas (Fig. 4), se podía controlar tanto la zona próxima a la Encañizada como el 
Mar Menor y la fachada Mediterránea de la Manga, incluida Isla Grosa, el cercano Islote del Farallón (Fig. 5) y el importante fondeadero del Estacio.

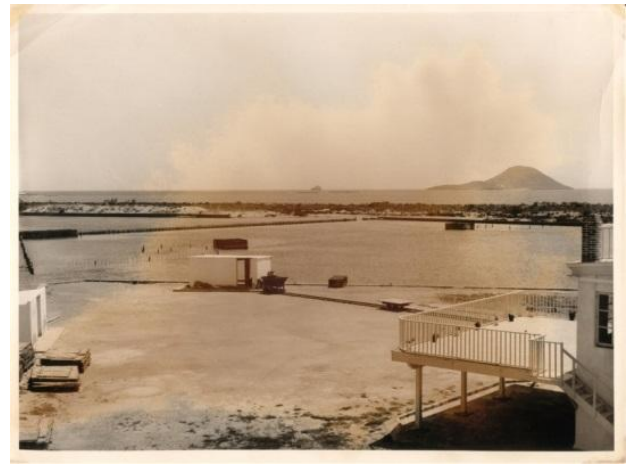

Fig.5- Fotografía de Isla Grosa tomada desde la localización de la Torre de la Encañizada. Tomada previamente al proceso urbanizador de la zona. Fuente: www.cabodepalosylamanga.com/fotos/

Todos estos motivos llevaron a la construcción de una torre de la que no han quedado vestigios arqueológicos visibles en superficie, pero que conocemos bien gracias a los planos conservados, las cartas e informes sobre su mantenimiento (Jiménez, 1981), e incluso una fotografía realizada en 1890 por el murciano Luis Federico Guirao Girada (Fig.6). En ella podemos apreciar con claridad la morfología de la torre: de construcción circular, con una escalera que daba acceso a la entrada elevada, restos de los enlucidos y un pretil reforzado en su cara exterior por ménsulas o contrafuertes que rodean la parte superior y que estarían destinados a soportar un matacán de madera que defendiera las piezas de artillería y los hombres emplazados en su cima. Estas estructuras, junto a la ausencia de ventanas, saeteras o troneras, proporcionarían un aspecto hermético de la torre.

Para el interior, los planos de Juan José Ordovás de 1799 son determinantes. Encontramos que esta estaba formada por dos pisos cubiertos por bóvedas, conectadas entre sí por una escalera de caracol que llegaba a la parte superior, cima en la que se refleja una especie de techumbre que formaría parte del matacán.

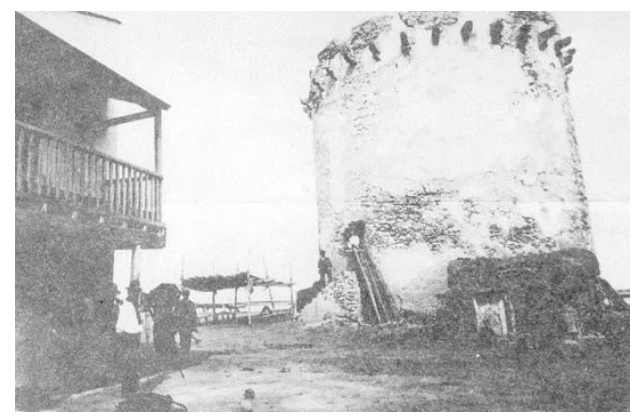

Fig. 6 - Torre de la Encañizada en 1890. Fotografía de Guirao Girada.

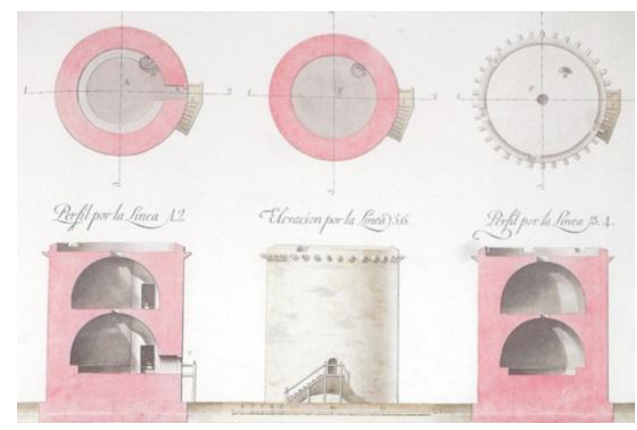

Fig. 7- Plano de la Torre de la Encañizada (Ordovás, 1799).

En cuanto a las medidas y dimensiones de la torre, la única información disponible se encuentra en el informe realizado con arreglo a la Orden del Excm. Sr. Ingeniero Militar del 9 de julio de 1869 , donde se apunta que la torre tiene 12 metros de diámetro en su base y una altura total de 13 metros. Medidas que, en caso de ser ciertas-pues es inverificable al no quedar vestigios arqueológicos en superficie y no haberse actuado sobre la zona-, demostrarían que el tamaño de la Torre de la Encañizada sería menor al de la Torre del Estacio.

La Torre de la Encañizada continuaría funcionando a lo largo de los siglos XVII y XVIII, reutilizándose una vez acabado su uso militar como almacén de los pescadores de la zona. Finalmente, en 1938 fue derribada para usar el material constructivo para rellenar dos tollos producidos por un temporal (Jiménez, 1981). 


\subsection{La Torre de San Miguel del Estacio: emblema de la defensa litoral}

Definida en el siglo XVIII como la mejor cosa que hay en nuestro Mediterráneo, con la cual está segura toda la costa (Rubio, 2000), la construcción de la Torre de San Miguel del Estacio comenzó a partir del año 1591, con un retraso total de 21 años desde su concepción en el plan del ingeniero Antonelli. No obstante, esta dilación en sus obras fue menor que la de otras torres previstas, pues su construcción apremiaba en cuanto a la importancia de su geoestrategia.

Así, encontramos cómo desde las ciudades de Murcia y Orihuela se acuerda construir antes esta torre que la del Pinatar, puesto que donde ay puerto y agua, y que serán allý los navíos de enemigos, y por donde todas las vezes que an trabucado a hazer mal y daño es por allý (Rubio, 2000). La existencia de un puerto y agua dulce nos remite a Isla Grosa, pero no debemos olvidar la importancia del propio fondeadero del Estacio que sería sin duda igualmente utilizado,

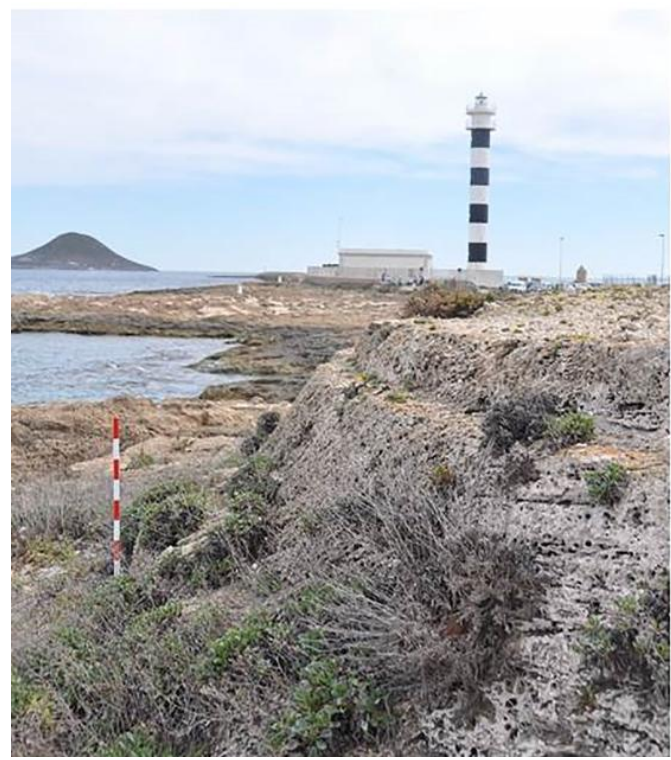

así como de la importante posición de control que ocuparía esta respecto al Mar Menor y a la fachada mediterránea (Fig. 4).

Para tales fines, se erigió una torre circular de unos 17,40 metros (Ordovás, 1799) y que conocemos sobradamente desde el punto de vista de las fuentes gracias a los informes de construcción y de reparaciones efectuados sobre ella y en los que no nos vamos a detener (Jiménez, 1981; Rubio, 2000). Destacar su estructura similar con la Torre de la Encañizada en cuanto a su interior -con dos pisos abovedados y escalera interior de caracol-, pero también las notables diferencias existentes entre ambas, principalmente el matacán y las troneras en piedra en su piso superior, lo que significa un mayor esfuerzo en recursos ya que la piedra había que traerla desde San Antón de Orihuela por ser la de La Manga blanda y poco resistente (Jiménez, 1981); su entrada sobreelevada a 10 metros (Rubio, 2000); y su basamento de planta circular y en tres nieveles (Fig. 9), único vestigio arqueológico que ha llegado hasta nuestros días.

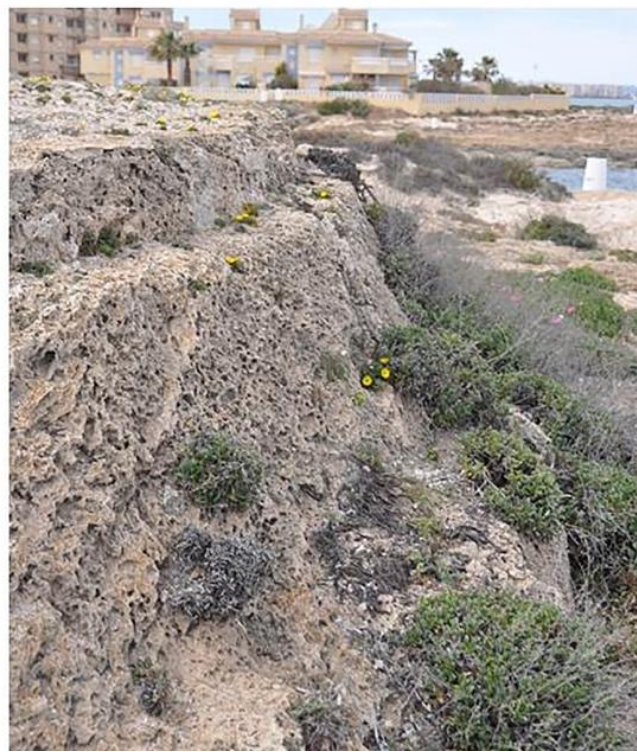

Fig. 8- Izquierda: Fotografía desde la cara noreste con Isla Grosa y el actual faro al fondo. Derecha: detalle de los tres niveles del basamento en su cara este. 


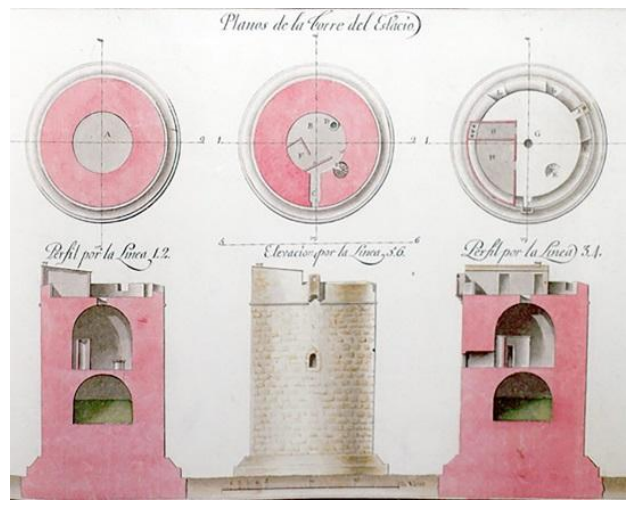

Fig. 9- Plano de la Torre de San Miguel del Estacio (Ordovás, 1799).

Con un diámetro estimado de 12,50 metros en su nivel superior, la cara mejor conservada es la este. Esta es la única que permite calcular con seguridad la altura del basamento, que alcanza los 1,55 metros de alto en la sucesión inclinada de tres niveles apreciables bajo la vegetación. No obstante, esta altura solo corresponde a esta parte de la estructura, pues estamos ante una construcción que se adapta a la topografía del afloramiento de piedra. Este es el caso de la cara oeste, en la que al encontrarse al nivel de la roca apenas se aprecia una obra clara del basamento como sí vemos en las caras norte, sur y este.

Por tanto, encontramos medidas similares entre las de ambas torres en sus basamentos aunque, la diferencia de altura entre una y otra debemos buscarla en el alzado. Mientrás que la Torre de la Encañizada tendría paredes rectas, en la del Estacio estas irían disminuyendo a medida que se elevaban, como indican las cartas de 1591 destinadas a su ejecución (Jiménez, 1981), permitiendo alcanzar esa altura citada de 17,40 metros. Si bien esta afirmación confronta con el plano del Ingeniero Ordovás, es posible que esta inclinación no fuera representada por su escasa apreciación, ya que no debía esta ser superior a media vara -0,41 m.- desde el suelo a los canes (Jiménez, 1981).

En relación al yacimiento, en la actualidad se observan restos cerámicos escasos con recubrimiento vidriado de clara adscripción a época moderna y surgen incógnitas en torno al basamento, pues en el nivel inferior se encuentran roturas y extracciones, al ser esta seguramente utilizada como cantera para la construcción del faro cercano, así como en la cara norte y oeste con estructuras ajenas a ese basamento circular pero poco comprensibles y cubiertas de vegetación (Fig. 10).

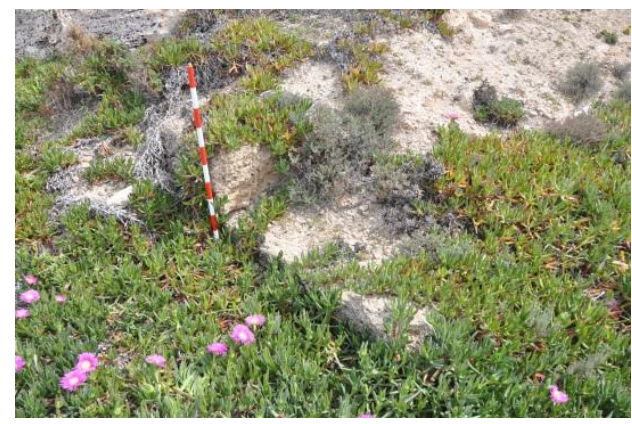

Fig. 10- Fotografía tomada en la cara noroeste. ¿Extracción de sillería? ¿Estructura aneja?

La Torre de San Miguel del Estacio continuó funcionando hasta bien entrado el siglo XIX como torre vigía defensiva, e incluso se llegó a plantear su uso como faro (Rubio, 2000), medida que no prosperó y que significó su fin, ya que para la construcción del faro actual a unos 160 metros al sur de la torre, se procedió al desmontaje de esta para la reutilización de su material constructivo, quedando sólo la base de aquella torre insignia de la costa del sureste peninsular.

\section{Conclusiones}

El objetivo de este trabajo era hacer una revisión de conjunto de la historia de las Torres de la Encañizada y de San Miguel del Estacio con el fin de hacer no sólo una revisión bibliográfica y documental de todo lo que sobre ellas se ha escrito, sino también abrir nuevas perspectivas de estudio y plantear hipótesis de trabajo con las que abordar de forma más completa el análisis histórico-arqueológico de lo que la construcción de estas torres supuso en el conjunto del Mar Menor y de la costa del sureste.

Se ha trabajado a partir de diferentes herramientas como las descripciones, los planos, mapas, cálculos SIG... sumando a esos resultados una fase de trabajo arqueológico de prospección y estudio de los vestigios 
arqueológicos visibles en superficie, testigos en superficie o bajo tierra que contribuyen a completar y mejorar esa lectura.

Estos resultados nos han permitido plantear nuevas propuestas de interpretación de la morfología de las torres, la importancia de su localización por razones diversas (defensa, vigilancia, seguridad, economía), la adaptación de estas al terreno o la existencia de restos arqueológicos aún por estudiar y preservar.

No obstante, queda mucho trabajo por hacer. Los archivos poseen abundante información sobre ambas torres, y muchas otras de la cuenca del Mar Menor necesitan una revisión con el fin de hacer un estudio completo a media escala que permita integrar este patrimonio en el lugar histórico que realmente ocuparon.

Un patrimonio que no se reduce a épocas moderna y contemporánea, sino que es un ejemplo de la buena ubicación que ocuparon las torres al ser pasos importantes en otros períodos históricos y defender tanto canales de agua como fondeaderos naturales utilizados desde épocas muy anteriores.

Además, planteamos la idoneidad de continuar con las excavaciones arqueológicas de estos lugares, puesto que serían campañas cortas al encontrar apenas unos metros de potencia arqueológica, pero que, sin duda, abrirían nuevas perspectivas de estudio para el futuro reciente de la investigación en este campo.

\section{Referencias}

Baragaño R., Alonso S. (1992). Gran Enciclopedia de la Región de Murcia. Tomo III. Ayalga Ediciones. Murcia. p. 320.

Berrocal M.C., Pérez J. (2010). "Puertos y fondeaderos de la costa murciana: dinámica costera, tipología de los asentamientos, interacciones económicas y culturales". in Bollettino di Archeologia on line. Direzione Generale Archeologia. Roma. pp. 36-50.

Cámara A. (1991). "Las torres del litoral en el reinado de Felipe II: una arquitectura para la defensa del territorio (y II)". in Espacio, tiempo y forma. Ed. UNED. Madrid. pp. 53-94.

Jiménez F. (1984). El municipio de San Javier en la Historia del Mar Menor. Academia Alfonso X El Sabio. Murcia. p. 322.

Lillo M. (1984). "Geomorfología litoral del Mar Menor". in Papeles del Departamento de Geografía. Universidad de Murcia - Servicio de Publicaciones. Murcia. pp. 9-48.

Martín-Consuegra G.J., Muñoz J.D., Abad J.M. (2009). La construcción territorial del Reino de Murcia en la Edad Moderna (ss. XVI-XVIII). Consejería de Educación, Formación y Empleo. Servicio de Publicaciones. Murcia. p. 70.

Ordovás J.J. (1799). Atlas político y militar del Reyno de Murcia formado por el capitán de ynfanteria e ingeniero ordinario de los Reales Ejércitos. Reedición Mimarq (2005). Murcia. p. 228.

Pérez-Ruzafa A., Marcos C., Pérez-Ruzafa J.M., Ros J.D. (1987). "Evolución de las características ambientales y de los poblamientos del Mar Menor (Murcia, SE de España)". in Anales de Biología. Universidad de Murcia - Secretariado de Publicaciones. Murcia. pp. 53-65.

Rubio J.M. (2000). Historia de las torres vigía de la costa del Reino de Murcia. Real Academia de Alfonso X el Sabio. Murcia. p. 201.

Ruiz J.J. (1995). Las dos caras de Jano: monarquía, ciudad e individuo. Servicio de Publicaciones. Murcia. p. 369.

Ruiz J.J. (1997). "La frontera de piedra: desarrollo de un sistema de defensa estático en la costa murciana (1588-1602)". in Actas del Congreso La Frontera Oriental Nazarí como sujeto histórico (s.XIII-XVI). Instituto de Estudios Almerienses. Almería. pp. 657-662. 